\title{
Free subgroups in certain generalized triangle groups of type $(2, m, 2)$
}

James Howie and Gerald Williams

March 21, 2006

\begin{abstract}
A generalized triangle group is a group that can be presented in the form $G=$ $\left\langle x, y \mid x^{p}=y^{q}=w(x, y)^{r}=1\right\rangle$ where $p, q, r \geq 2$ and $w(x, y)$ is a cyclically reduced word of length at least 2 in the free product $\mathbb{Z}_{p} * \mathbb{Z}_{q}=\left\langle x, y \mid x^{p}=y^{q}=1\right\rangle$. Rosenberger has conjectured that every generalized triangle group $G$ satisfies the Tits alternative. It is known that the conjecture holds except possibly when the triple $(p, q, r)$ is one of $(3,3,2),(3,4,2),(3,5,2)$, or $(2, m, 2)$ where $m=3,4,5,6,10,12,15,20,30,60$. In this paper we show that the Tits alternative holds in the cases $(p, q, r)=(2, m, 2)$ where $m=6,10,12,15,20,30,60$.
\end{abstract}

\section{Introduction}

A generalized triangle group is a group that can be presented in the form

$$
G=\left\langle x, y \mid x^{p}=y^{q}=w(x, y)^{r}=1\right\rangle
$$

where $p, q, r \geq 2$ and $w(x, y)$ is a cyclically reduced word of length at least 2 in the free product $\mathbb{Z}_{p} * \mathbb{Z}_{q}=\left\langle x, y \mid x^{p}=y^{q}=1\right\rangle$ that is not a proper power. It was conjectured by Rosenberger [15] that every generalized triangle group $G$ satisfies the Tits alternative. That is, $G$ either contains a non-abelian free subgroup or has a soluble subgroup of finite index.

It is now known that the Tits alternative holds for a generalized triangle group $G$ except possibly when the triple $(p, q, r)$ is one of $(3,3,2),(3,4,2),(3,5,2)$, or $(2, m, 2)$ where $m \geq 3$. (See [9] for a survey of these results.) In recent work Benyash-Krivets [3, $4]$ considers the case $(2, m, 2)$. He has shown that if $m \geq 7, m \neq 10,12,15,20,30,60$ then the Tits alternative holds for $G$. In this paper we augment that result to prove the following:

Main Theorem. Let $G=\left\langle x, y \mid x^{2}=y^{m}=w(x, y)^{2}=1\right\rangle$ where $w(x, y)=$ $x y^{\alpha_{1}} \ldots x y^{\alpha_{k}}, 1 \leq \alpha_{i}<m, m \geq 6$. Then the Tits alternative holds for $G$.

If $k=1$ then the Tits alternative holds for $G$ by [8]. If $m=6$ and $k=2$ or 3 then the Tits alternative holds for $G$ by $[15,14]$ respectively. The Main Theorem then follows from Theorems 1, 2 and 3: 
Theorem 1 Let $G$ be as defined in the Main Theorem. If $m=6$ and $k>3$, then $G$ contains a non-abelian free subgroup.

Theorem 2 Let $G$ be as defined in the Main Theorem. If $m=5 p$ where $p \neq 5$ is prime and $k>1$, then $G$ contains a non-abelian free subgroup.

Theorem 3 Let $G$ be as defined in the Main Theorem. If $k>1$ and $m=12,20,30$, or 60 then $G$ contains a non-abelian free subgroup.

Theorem 1 has independently been obtained by Barkovich and Benyash-Krivets [1, $5]$, and for this reason we do not give a complete proof. However, we require Theorem 1 in an essential way in the proofs of the other results, so in order to make our paper self-contained we have included a sketch proof in an Appendix.

\section{Preliminaries}

We first recall some definitions and well-known facts concerning generalized triangle groups; further details are available in (for example) [9]. Let $G$ be as defined in the Main Theorem, but with $m \geq 3$. A homomorphism $\rho: G \rightarrow H$ (for some group $H$ ) is said to be essential if $\rho(x), \rho(y), \rho(w)$ are of orders $2, m, 2$ respectively. By [2] $G$ admits an essential representation into $P S L(2, \mathbb{C})$.

A projective matrix $A \in P S L(2, \mathbb{C})$ is of order $n$ if and only if $\operatorname{tr}(A)=2 \cos (q \pi / n)$ for some $(q, n)=1$. Note that in $\operatorname{PSL}(2, \mathbb{C})$ traces are only defined up to sign. A subgroup of $P S L(2, \mathbb{C})$ is said to be elementary if it has a soluble subgroup of finite index, and is said to be non-elementary otherwise.

Let $\rho:\left\langle x, y \mid x^{2}=y^{m}=1\right\rangle \rightarrow P S L(2, \mathbb{C})$ be given by $x \mapsto X, y \mapsto Y$ where $X, Y$ have orders $2, m$, respectively. Then $w(x, y) \mapsto w(X, Y)$. By Horowitz [12] $\operatorname{tr} w(X, Y)$ is a polynomial with rational coefficients in $\operatorname{tr} X, \operatorname{tr} Y, \lambda:=\operatorname{tr} X Y$, of degree $k$ in $\lambda$. Since $X, Y$ have orders $2, m$, respectively, we may assume (by composing $\rho$ with an automorphism of $\left\langle x, y \mid x^{2}=y^{m}=1\right\rangle$ if necessary), that $\operatorname{tr} X=0, \operatorname{tr} Y=2 \cos (\pi / m)$. Moreover (again by [12]) $X$ and $Y$ can be any elements of $P S L(2, \mathbb{C}$ ) with these traces. Suppressing $\operatorname{tr} X, \operatorname{tr} Y$ in the notation we define the trace polynomial of $G$ to be $\tau(\lambda):=\operatorname{tr} w(X, Y)$.

The representation $\rho$ induces an essential representation $G \rightarrow P S L(2, \mathbb{C})$ if and only if $\operatorname{tr} \rho(w)=0$; that is, if and only if $\lambda$ is a root of $\tau$. Note that $\tau(\lambda)= \pm \tau(-\lambda)$ so the roots $\lambda,-\lambda$ occur with equal multiplicity.

By [12] the leading coefficient of $\tau$ is given by

$$
c=\frac{1}{(\sin (\pi / m))^{k}} \prod_{i=1}^{k} \sin \left(\frac{\pi \alpha_{i}}{m}\right) .
$$

(This expression can also be obtained from Lemma 12 in the Appendix, where we obtain a formula for each of the coefficients of $\tau$.) For each $1 \leq j \leq m / 2$ we shall let $t_{j}=\sin (j \pi / m)$ and let $k_{j}$ denote the number of times $\alpha_{i}=j$ or $\alpha_{i}=(m-j)$ in 
the word $w(x, y)$ (so that $k=k_{1}+\ldots+k_{\lfloor m / 2\rfloor}$ ). The above formula then becomes $c=\left(t_{1}^{k_{1}} \ldots t_{\lfloor m / 2\rfloor}^{k_{\lfloor m / 2\rfloor}}\right) /\left(\sin (\pi / m)^{k}\right)$.

Now if $X, Y$ generate a non-elementary subgroup of $P S L(2, \mathbb{C})$ then $\rho(G)$ (and hence $G$ ) contains a non-abelian free subgroup. Thus in proving that $G$ contains a non-abelian free subgroup we may assume that $X, Y$ generate an elementary subgroup of $P S L(2, \mathbb{C})$. By Corollary 2.4 of [15] there are then three possibilities: (i) $X, Y$ generate a finite subgroup of $P S L(2, \mathbb{C}$ ); (ii) $\operatorname{tr}[X, Y]=2$; or (iii) $\operatorname{tr} X Y=0$.

The finite subgroups of $P S L(2, \mathbb{C})$ are the alternating groups $A_{4}$ and $A_{5}$, the symmetric group $S_{4}$, cyclic and dihedral groups (see for example [7]). Manipulation using trace identities shows that (ii) is equivalent to $\operatorname{tr} X Y= \pm \sin (\pi / m)$. These values occur as roots of $\tau$ if and only if $G$ admits an essential cyclic representation. Such a representation can be realized as $x \mapsto A, y \mapsto B$ where

$$
A=\left(\begin{array}{cc}
e^{i \pi / 2} & 0 \\
0 & e^{-i \pi / 2}
\end{array}\right), \quad B=\left(\begin{array}{cc}
e^{i \pi / m} & 0 \\
0 & e^{-i \pi / m}
\end{array}\right) .
$$

In case (iii) $X$ and $Y$ generate the finite dihedral group $D_{2 m}$. We summarize the above as

Lemma 4 Let $G$ be as defined in the Main Theorem, with $m \geq 3$. Suppose $G \rightarrow$ $P S L(2, \mathbb{C})$ is an essential representation given by $x \mapsto X, y \mapsto Y$, where $\operatorname{tr} X=$ $0, \operatorname{tr} Y=2 \cos (\pi / m)$. If $G$ does not contain a non-abelian free subgroup then one of the following occurs:

1. $X, Y$ generate $A_{4}, S_{4}$, or $A_{5}$;

2. $\operatorname{tr} X Y= \pm 2 \sin (\pi / m)$;

3. $\operatorname{tr} X Y=0$ and $\langle X, Y\rangle \cong D_{2 m}$.

Case (2) occurs if and only if $G$ admits an essential cyclic representation.

Remark 5 If $X, Y$ generate $A_{4}$ then $m=3$ and $X Y$ has order 3 , so $\operatorname{tr} X Y= \pm 1$. If $X, Y$ generate $S_{4}$ then either (a) $m=3$ and $X Y$ has order 4 , so $\operatorname{tr} X Y= \pm \sqrt{2}$; or (b) $m=4$ and $X Y$ has order 3 , so $\operatorname{tr} X Y= \pm 1$. If $X, Y$ generate $A_{5}$ then either (a) $m=3$ and $X Y$ has order 5 ; or (b) $m=5$ and $X Y$ has order 3, so $\operatorname{tr} X Y= \pm 1$; or (c) $m=5$ and $X Y$ has order 5 , in which case $X Y$ is conjugate to $Y^{2}$ so $\operatorname{tr} X Y= \pm \operatorname{tr} Y^{2}= \pm\left((\operatorname{tr} Y)^{2}-2\right)$.

\section{The case $m=4$}

Lemma 6 Let $G=\left\langle x, y \mid x^{2}=y^{4}=\left(x y^{\alpha_{1}} \ldots x y^{\alpha_{k}}\right)^{2}=1\right\rangle$ and let $k_{2}$ denote the number of values of $i$ for which $\alpha_{i}=2$. Then $G$ contains a non-abelian free subgroup unless one of the following holds:

1. $k$ is odd and one of the following holds: 
(a) $\sum_{i=1}^{k} \alpha_{i}=0 \bmod 4$;

(b) $\sum_{i=1}^{k} \alpha_{i}=2 \bmod 4$ and $k_{2}=1$;

(c) $\sum_{i=1}^{k} \alpha_{i}=1,3 \bmod 4$ and $k_{2}=0$;

2. $k$ is even and one of the following holds:

(a) $\sum_{i=1}^{k} \alpha_{i}=2 \bmod 4$;

(b) $\sum_{i=1}^{k} \alpha_{i}=0 \bmod 4$ and either

(i). $k_{2}=0$ and $k=2 \bmod 4$; or

(ii). $k_{2}=2$;

(c) $\sum_{i=1}^{k} \alpha_{i}=1,3 \bmod 4$ and $k_{2}=1$.

\section{Proof}

By Lemma 4 and Remark 5 we may assume that the roots of the trace polynomial $\tau$ are among $\pm \sqrt{2}, 0, \pm 1$. Thus

$$
\tau(\lambda)=c \lambda^{s}\left(\lambda^{2}-1\right)^{t}\left(\lambda^{2}-2\right)^{u}
$$

where $s+2 t+2 u=k$ and

$$
c=\frac{1}{(\sin (\pi / 4))^{k}}(\sin (\pi / 4))^{k_{1}}(\sin (2 \pi / 4))^{k_{2}}=\sqrt{2}^{k_{2}},
$$

where $k_{1}, k_{2}$ denote the number of times $\alpha_{i}$ takes the values $\pm 1,2$ respectively. (Note that $k$ and $s$ are of the same parity.)

Let

$$
A=\left(\begin{array}{cc}
i & 0 \\
1 & -i
\end{array}\right), \quad B=\left(\begin{array}{cc}
(1+i) / \sqrt{2} & z \\
0 & (1-i) / \sqrt{2}
\end{array}\right)
$$

be elements of $P S L(2, \mathbb{C})$ so that $\operatorname{tr} A=0, \operatorname{tr} B=\sqrt{2}, \operatorname{tr} A B=z-\sqrt{2}$. Consider the representation $\rho:\left\langle x, y \mid x^{2}=y^{4}=1\right\rangle \rightarrow P S L(2, \mathbb{C})$ given by $x \mapsto A, y \mapsto B$ then

$$
\begin{aligned}
\operatorname{tr} \rho\left(x y^{\alpha_{1}} \ldots x y^{\alpha_{k}}\right) & =\tau(z-\sqrt{2}) \\
& = \pm(\sqrt{2})^{k_{2}}(z-\sqrt{2})^{s}\left(z^{2}-2 \sqrt{2} z+1\right)^{t}(z-2 \sqrt{2})^{u} z^{u}
\end{aligned}
$$

whose constant term is 0 if $u>0$, and $\pm(\sqrt{2})^{k_{2}+s}$ if $u=0$. Now the constant term in $\operatorname{tr}\left(A B^{\alpha_{1}} \ldots A B^{\alpha_{k}}\right)$ is given by $2 \cos \left(\left(2 k+\sum_{i=1}^{k} \alpha_{i}\right) \pi / 4\right) \in\{ \pm 2, \pm \sqrt{2}\}$. If $u>0$ we have that $2 k+\sum_{i=1}^{k} \alpha_{i}=2 \bmod 4$, and one of the conclusions 1 (a) or 2(a) holds. Thus we may assume $u=0$, and therefore $k_{2}+s=1$ or 2 .

Suppose $k$ is odd. Then $s$ is odd. Since $2 k+\sum_{i=1}^{k} \alpha_{i} \neq 2 \bmod 4$ we have $\sum_{i=1}^{k} \alpha_{i}=1,2$, or $3 \bmod 4$. If $\sum_{i=1}^{k} \alpha_{i}=2 \bmod 4$ then $k_{2}$ is odd so $k_{2}=1, s=1$ and we are in case $1(\mathrm{~b})$. If $\sum_{i=1}^{k} \alpha_{i}=1,3 \bmod 4$ then $k_{2}$ is even so $k_{2}=0, s=1$ and we are in case $1(\mathrm{c})$.

Suppose $k$ is even. Then $s$ is even. Since $2 k+\sum_{i=1}^{k} \alpha_{i} \neq 2 \bmod 4$ we have $\sum_{i=1}^{k} \alpha_{i}=0,1$, or $3 \bmod 4$. If $\sum_{i=1}^{k} \alpha_{i}=1$ or $3 \bmod 4$ then $k_{2}$ is odd so $k_{2}=1, s=0$ 
and we are in case 2(c). If $\sum_{i=1}^{k} \alpha_{i}=0 \bmod 4$ then $k_{2}$ is even so either $k_{2}=0, s=2$ or $k_{2}=2, s=0$. In the latter option we are in case 2(b)(ii). In the former 0 is a root of $\tau(\lambda)$ so $G$ admits an essential dihedral representation. Thus $\sum_{i=1}^{k}(-1)^{i} \alpha_{i}=2$ $\bmod 4$. Combining this with $\sum_{i=1}^{k} \alpha_{i}=0 \bmod 4$ and the fact that each $\alpha_{i}$ is odd, we obtain $k=2 \bmod 4$ and we are in case $2(\mathrm{~b})(\mathrm{i})$.

\section{The cases $m=10,15$}

In this section we consider the following situation. Let $G$ be as defined in the Main Theorem where $m=5 p$ for some prime $p$. We first consider the case where $k$ is even.

Lemma 7 Let $G$ be as defined in the Main Theorem, where $m=5 p$ for some prime $p$ and where $k$ is even. Then $G$ contains a non-abelian free subgroup.

\section{Proof}

If $p=2$ then $G$ contains a non-abelian free subgroup by [16, Theorem A]. Suppose then that $p$ is odd.

Consider a homomorphism $\theta: G \rightarrow \mathbb{Z}_{10 p} \cong \mathbb{Z}_{2} \times \mathbb{Z}_{5 p}$ such that $\theta(x), \theta(y)$ have orders $2,5 p$ respectively. Then, up to an automorphism of $\mathbb{Z}_{10 p}$ we may assume that $\theta(x)=5 p, \theta(y)=2$. Then $\theta(w)=5 p k+2 \sum_{i=1}^{k} \alpha_{i}$, which is not of order 2 , since $k$ is even and $p$ is odd. Hence we must have $\theta(w)=0$, so $\theta$ is not essential.

In a similar way, consider a homomorphism $\theta: G \rightarrow\left\langle a, b \mid a^{2}=b^{5 p}=(a b)^{2}=1\right\rangle \cong$ $D_{10 p}$ such that $\theta(x), \theta(y)$ have orders $2,5 p$ respectively. Then, up to an automorphism of $D_{10 p}$ we may assume that $\theta(x)=a, \theta(y)=b$. Then $\theta(w)=b^{\sum_{i=1}^{k}(-1)^{i} \alpha_{i}}$, which is not of order 2, since $p$ is odd. Hence we must have $\theta(w)=1$, so $\theta$ is not essential.

Thus $G$ admits no essential cyclic or dihedral representation, so (since we also have $m>5$ ) Lemma 4 implies that $G$ contains a non-abelian free subgroup.

By Lemma 7 we may restrict attention to the case where $k$ is odd. We do so throughout the remainder of this section without further comment.

Now $G$ maps homomorphically onto the group

$$
\bar{G}=\left\langle x, y \mid x^{2}=y^{5}=\bar{w}(x, y)^{2}=1\right\rangle
$$

where $\bar{w} \in\left\langle x, y \mid x^{2}=y^{5}=1\right\rangle$ is given by $\bar{w}=x y^{\beta_{1}} \ldots x y^{\beta_{k}}$ where $\beta_{i}=\alpha_{i} \bmod 5$ $(1 \leq i \leq k)$. Now $\bar{w} \neq y^{\beta}$ for any $\beta$, since $k$ is odd. If $\bar{w}=x$ then $\bar{G} \cong \mathbb{Z}_{2} * \mathbb{Z}_{5}$ and so $\bar{G}$, and hence $G$, contains a non-abelian free subgroup. If $\bar{w}$ is a proper power then $\bar{G}$, and hence $G$, contains a non-abelian free subgroup by [2].

Thus we will assume that $\bar{w}$ can be freely reduced to a word of the form $\bar{w}=$ $x y^{\gamma_{1}} \ldots x y^{\gamma_{\ell}}$ that is not a proper power, where $1 \leq \gamma_{i} \leq 4(1 \leq i \leq \ell), \ell \geq 1$. Hence the corresponding presentation (1) is a presentation of $\bar{G}$ as a generalized triangle group. We let $\tau(\lambda), \sigma(\mu)$ denote the trace polynomials of $G$ and $\bar{G}$ respectively.

Lemma 8 If 1 is a repeated root of $\sigma(\mu)$ then $G$ contains a non-abelian free subgroup. 


\section{Proof}

Let $q: G \rightarrow \bar{G}$ denote the canonical epimorphism. By hypothesis, there is an essential representation $\rho: \bar{G} \rightarrow P S L_{2}\left(\mathbb{C}[\mu] /(\mu-1)^{2}\right)$. Indeed, we can construct $\rho$ explicitly via:

$$
\rho(x)=\left(\begin{array}{cc}
0 & -1 \\
1 & 0
\end{array}\right), \quad \rho(y)=\left(\begin{array}{cc}
e^{i \pi / 5} & \mu \\
0 & e^{-i \pi / 5}
\end{array}\right) .
$$

Composing this with the canonical epimorphism

$$
\psi: P S L_{2}\left(\mathbb{C}[\mu] /(\mu-1)^{2}\right) \rightarrow P S L_{2}(\mathbb{C}[\mu] /(\mu-1)) \cong P S L_{2}(\mathbb{C})
$$

gives an essential representation $\tilde{\rho}=\psi \circ \rho: \bar{G} \rightarrow P S L_{2}(\mathbb{C})$ with image $A_{5}$, corresponding to the root 1 of the trace polynomial.

Let $\bar{K}$ denote the kernel of $\tilde{\rho}, V$ the kernel of $\psi$, and $K$ the kernel of the composite map $\tilde{\rho} \circ q: G \rightarrow P S L_{2}(\mathbb{C})$. Then $V$ is a complex vector space, since its elements have the form $\pm(I+(\mu-1) A)$ for various $2 \times 2$ matrices $A$, with multiplication

$$
[ \pm(I+(\mu-1) A)][ \pm(I+(\mu-1) B)]= \pm(I+(\mu-1)(A+B)) .
$$

Our strategy is to apply the techniques of [13] to $K$ to obtain the existence of a non-abelian free subgroup. To this end we will first analyse the structure of $V \supset \rho(\bar{K})=\rho(q(K))$ to obtain a large free abelian quotient $K / N$ of $K$ with suitable properties. We will then exhibit $K$ as the fundamental group of a certain CWcomplex $X$, and show that the second homology group of the covering complex of $X$ corresponding to $N$ has a free $\mathbb{Z}(K / N)$-submodule of large rank.

Now $\bar{K}$ is generated by conjugates of $(x y)^{3}$. Consider four such conjugates: $c_{1}=$ $(x y)^{3}, c_{2}=x(x y)^{3} x, c_{3}=y x y^{3}(x y)^{3} y^{2} x y^{4}$, and $c_{4}=y x y^{4}(x y)^{3} y x y^{4}$. A calculation shows that $\rho\left(c_{i}\right)= \pm\left(I+(\mu-1) M_{i}\right)$ where

$M_{1}=\left(\begin{array}{cc}-1 & z_{1} \\ -\bar{z}_{1} & 1\end{array}\right), \quad M_{2}=\left(\begin{array}{cc}1 & \bar{z}_{1} \\ -z_{1} & -1\end{array}\right), \quad M_{3}=\left(\begin{array}{cc}z_{2} & -z_{3} \\ -z_{3} & -z_{2}\end{array}\right), \quad M_{4}=\left(\begin{array}{cc}\bar{z}_{2} & \bar{z}_{3} \\ \bar{z}_{3} & -\bar{z}_{2}\end{array}\right)$,

where

$$
\begin{aligned}
& z_{1}=\frac{-(1+\sqrt{5})}{2}+i \frac{\sqrt{10-2 \sqrt{5}}}{2}, \\
& z_{2}=\frac{3+\sqrt{5}}{2}+i \frac{\sqrt{10-2 \sqrt{5}}}{2}, \\
& z_{3}=-1+i \frac{(3+\sqrt{5}) \sqrt{10-2 \sqrt{5}}}{4} .
\end{aligned}
$$

By considering (for example) the upper right hand entries, it is easy to verify that $M_{1}, M_{2}, M_{3}, M_{4}$ are linearly independent over $\mathbb{Q}$. The group $A_{5}$ acts on $V$ via conjugation and since $\tilde{\rho}(x)$ is of order 2, the action of $\tilde{\rho}(x)$ on $V$ is diagonalizable. Moreover, the only possible eigenvalues are \pm 1 . Thus $V$ splits as a $\mathbb{Q}$-direct sum $V_{+} \oplus V_{-}$, where $\tilde{\rho}(x)$ acts as the identity on $V_{+}$and as the antipodal map $v \mapsto-v$ on $V_{-}$. The canonical projection $V \rightarrow V_{-}$with kernel $V_{+}$is $\tilde{\rho}(x)$-equivariant. 
For $j=3,4$, the off-diagonal entries of $M_{j}$ are equal. It follows easily that $\rho\left(x c_{j}\right)$ has trace 0 , so is of order 2 , and hence $\rho\left(x c_{j} x\right)=\rho\left(c_{j}^{-1}\right)$. Note also that $x c_{1} x=c_{2}$ and $x c_{2} x=c_{1}$. Thus $\rho\left(c_{1} c_{2}^{-1}\right), \rho\left(c_{3}\right), \rho\left(c_{4}\right) \in V_{-}$and $\rho\left(c_{1} c_{2}\right) \in V_{+}$. Let $N$ be the pre-image of $V_{+}$in $K$. Then $N$ is normal in $K$ and is invariant under conjugation by $x$. It follows that $K / N$ is free abelian of rank at least 3 and that $\tilde{\rho}(x)$ acts on $K / N$ as the antipodal map.

Note that $K$ is the fundamental group of a 2-dimensional CW-complex $X$ arising from the given presentation of $G$. This complex $X$ has 60 cells of dimension 0,120 cells of dimension 1 , and $60\left(\frac{1}{2}+\frac{1}{5}+\frac{1}{2}\right)=72$ cells of dimension 2 . Here, $60 / 5=12$ of the 2-cells (call them $\alpha_{1}, \ldots, \alpha_{12}$, say) arise from the relator $y^{5 p}, 60 / 2=30\left(\alpha_{13}, \ldots, \alpha_{42}\right.$, say) arise from the relator $x^{2}$, and $60 / 2=30\left(\alpha_{43}, \ldots, \alpha_{72}\right.$, say $)$ arise from the relator $w(x, y)^{2}$. Moreover, $\alpha_{1}, \ldots, \alpha_{12}$ are attached by maps which are $p$ th powers. Let $\widehat{X}$ be the regular covering complex of $X$ corresponding to the normal subgroup $N$ of $K$ and let $\widehat{\alpha}_{i}$ denote a lift of the 2-cell $\alpha_{i}$. Then each of $\widehat{\alpha}_{1}, \ldots, \widehat{\alpha}_{12}$ is a 2-cell attached by a map which is a $p$ th power.

Let $G F_{p}$ denote the field with $p$ elements. Now $H_{2}\left(\widehat{X}, G F_{p}\right)$ is a subgroup of the 2-chain group $C_{2}\left(\widehat{X}, G F_{p}\right)$ and since $K / N$ freely permutes the cells of $\widehat{X}, C_{2}\left(\widehat{X}, G F_{p}\right)$ is a free $G F_{p}(K / N)$-module on the basis $\widehat{\alpha}_{1}, \ldots, \widehat{\alpha}_{72}$. Let $Q$ be the free $G F_{p}(K / N)$ submodule of $C_{2}\left(\widehat{X}, G F_{p}\right)$ of rank 12 generated by $\widehat{\alpha}_{1}, \ldots, \widehat{\alpha}_{12}$. Since these 2-cells are attached by maps which are $p$ th powers, their boundaries in the 1-chain group $C_{1}\left(\widehat{X}, G F_{p}\right)$ are zero. Thus $Q$ is a subgroup of $H_{2}\left(\widehat{X}, G F_{p}\right)$.

Suppose $Q \neq H_{2}\left(\widehat{X}, G F_{p}\right)$, and let $\widehat{\beta} \in H_{2}\left(\widehat{X}, G F_{p}\right) \backslash Q$. Then $\widehat{\beta}=\sum_{i=1}^{72} \mu_{i} \widehat{\alpha}_{i}$ where $\mu_{i} \in G F_{p}(K / N)(1 \leq i \leq 72)$ and $\mu_{q} \neq 0$ for some $q>12$. Let $L$ be the submodule of $H_{2}\left(\widehat{X}, G F_{p}\right)$ generated by $\widehat{\alpha}_{1}, \ldots, \widehat{\alpha}_{12}, \widehat{\beta}$. Let $\pi_{q}: C_{2}\left(\widehat{X}, G F_{p}\right) \rightarrow$ $G F_{p}(K / N)$ denote the projection map on the basis element $\widehat{\alpha}_{q}$ and suppose $\lambda, \lambda_{1}, \ldots, \lambda_{12} \in G F_{p}(K / N)$ satisfy

$$
v:=\lambda \widehat{\beta}+\lambda_{1} \widehat{\alpha}_{1}+\ldots+\lambda_{12} \widehat{\alpha}_{12}=0
$$

in $C_{2}\left(\widehat{X}, G F_{p}\right)$. Then $0=\pi_{q}(v)=\lambda \mu_{q}$, and since $G F_{p}(K / N)$ is an integral domain we have that $\lambda=0$ so $\lambda_{1} \widehat{\alpha}_{1}+\ldots+\lambda_{12} \widehat{\alpha}_{12}=0$ in $Q$. But $\widehat{\alpha}_{1}, \ldots, \widehat{\alpha}_{12}$ form a $G F_{p}(K / N)$ basis for $Q$ so $\lambda_{1}=\cdots=\lambda_{12}=0$ and hence $L$ is free on $\left\{\widehat{\alpha}_{1}, \ldots, \widehat{\alpha}_{12}, \widehat{\beta}\right\}$. Thus $H_{2}\left(\widehat{X}, G F_{p}\right)$ contains a free $G F_{p}(K / N)$-submodule of rank $13=1+\chi(X)$ so by $[13$, Proposition 2.1 and Theorem 2.2], $K=\pi_{1}(X)$ contains a non-abelian free subgroup.

Suppose then that $H_{2}\left(\widehat{X}, G F_{p}\right)=Q$. We argue as in the proof of $[13$, Corollary 3.2]. The element $c_{1} c_{2} \in N$ is mapped to the element $\pm\left(I+(\mu-1)\left(M_{1}+M_{2}\right)\right)$ of infinite order in $V_{+}$so $N^{a b}$ has torsion-free rank at least 1 . Thus $H_{1}\left(\widehat{X}, G F_{p}\right) \cong$ $N^{a b} / p N^{a b} \neq 0$. We also have that $H_{2}\left(\widehat{X}, G F_{p}\right)$ is a free $G F_{p}(K / N)$-module and $K / N$ is a free abelian group of rank at least 3 , so by [13, Theorem D] there is a subgroup $J / N$ of $K / N$ such that $(K / N) /(J / N) \cong K / J \cong \mathbb{Z}^{2}$ and $H_{1}\left(\widehat{X}, G F_{p}\right)$ contains a nonzero free $G F_{p}(J / N)$-submodule. Moreover, $J / N$ is infinite so this module is of infinite $G F_{p}$-dimension.

Thus, by definition, the Bieri-Strebel invariant $([6]) \Sigma$ of the $G F_{p}(K / N)$-module $H_{1}\left(\widehat{X}, G F_{p}\right)$ is a proper subset of the sphere $S^{d-1}$ (where $d$ is the rank of the free 
abelian group $K / N)$. But $\Sigma=-\Sigma$, since $\tilde{\rho}(x)$ acts as the antipodal map on $K / N$. Hence $\Sigma \cup-\Sigma \neq S^{d-1}$, and so $N$ has a non-abelian free subgroup by [6, Theorem 4.1].

Lemma 9 If $\bar{G}$ has an essential cyclic representation then $G$ contains a non-abelian free subgroup.

\section{Proof}

Let $q: G \rightarrow \bar{G}$ denote the canonical epimorphism. Since $\bar{G}$ admits an essential cyclic representation, $\pm 2 \sin (\pi / 5)$ are roots of its trace polynomial, so there also exists an essential representation $\rho: \bar{G} \rightarrow P S L(2, \mathbb{C})$ given by $x \mapsto X, y \mapsto Y$, where

$$
X=\left(\begin{array}{cc}
i & 1 \\
0 & -i
\end{array}\right), \quad Y=\left(\begin{array}{cc}
e^{i \pi / 5} & 0 \\
0 & e^{-i \pi / 5}
\end{array}\right)
$$

Let $\psi: \rho(\bar{G}) \rightarrow P S L(2, \mathbb{C})$ be given by

$$
X \mapsto\left(\begin{array}{cc}
i & 0 \\
0 & -i
\end{array}\right), \quad Y \mapsto Y
$$

then $\tilde{\rho}:=\psi \circ \rho: \bar{G} \rightarrow P S L(2, \mathbb{C})$ is an essential representation with image $\mathbb{Z}_{10}$. Let $K, \bar{K}, \bar{N}$ denote the kernels of the maps $\tilde{\rho} \circ q, \tilde{\rho}, \rho$, respectively. Then $\bar{K}$ is generated by $c_{t}:=y^{t} x y^{-t} x(t=1,2,3,4)$. Now for each $t$

$$
\rho\left(c_{t}\right)=\left(\begin{array}{cc}
1 & i\left(e^{2 \pi t i / 5}+1\right) \\
0 & 1
\end{array}\right)
$$

so $\rho\left(c_{1}\right), \rho\left(c_{2}\right), \rho\left(c_{3}\right), \rho\left(c_{4}\right)$ are linearly independent over $\mathbb{Q}$ and hence $\rho(\bar{K}) \cong \mathbb{Z}^{4}$. Thus $\bar{G} / \bar{K} \cong \mathbb{Z}_{10}$ and $\bar{K} / \bar{N} \cong \mathbb{Z}^{4}$, so if $N$ denotes the preimage of $\bar{N}$ in $G$ then $N \triangleleft K \triangleleft G$ and $G / K \cong \mathbb{Z}_{10}, K / N \cong \mathbb{Z}^{4}$. Moreover, $x c_{t} x=c_{t}^{-1}$ for each $t$ so $\tilde{\rho}(x)$ acts as the antipodal map on $K / N$.

Now $K$ is the fundamental group of a 2-dimensional CW-complex with 10 0-cells, 20 1-cells and 122 -cells, 2 of which correspond to the relator $y^{5 p}$, and so are attached by $p$ th powers. The argument given in the proof of Lemma 8 then shows that $K$ has a non-abelian free subgroup.

For the following lemma, recall that $2 \ell$ is the (free product) length of $\bar{w}(x, y)$ and that $\sigma(\mu)$ denotes the trace polynomial of $\bar{G}$.

Lemma 10 Suppose that $\ell$ is odd and that $\bar{G}$ admits no essential cyclic representation. If 0 is a repeated root of $\sigma(\mu)$ then $\bar{G}$ (and hence $G$ ) contains a non-abelian free subgroup.

\section{Proof}

Let $\eta=2 \cos (\pi / 5)=(1+\sqrt{5}) / 2$ and note that $\eta^{4}-3 \eta^{2}+1=0$. By Lemma 4 and 
Remark 5 we may assume that the roots of $\sigma$ are among $\pm\left(\eta^{2}-2\right)= \pm \eta^{ \pm 1}, \pm 1$, $\pm 2 \sin (\pi / 5)= \pm \sqrt{4-\eta^{2}}, 0$. The leading coefficient of $\sigma(\mu)$ is given by $c=\eta^{k_{2}}$. Thus $\sigma(\mu)$ takes the form

$$
\sigma(\mu)=\eta^{k_{2}} \mu^{s}\left(\mu^{2}-1\right)^{t}\left(\mu^{2}-\eta^{-2}\right)^{u}\left(\mu^{2}-\left(4-\eta^{2}\right)\right)^{v}
$$

where $s+2 t+2 u+2 v=\ell$. Let $A, B \in P S L(2, \mathbb{C})$ be defined as follows:

$$
A=\left(\begin{array}{cc}
i & 0 \\
1 & -i
\end{array}\right), \quad B=\left(\begin{array}{cc}
e^{i \pi / 5} & z \\
0 & e^{-i \pi / 5}
\end{array}\right) .
$$

Then $\operatorname{tr} A=0, \operatorname{tr} B=\eta, \operatorname{tr} A B=z-\sqrt{4-\eta^{2}}$.

Consider the representation $\rho:\left\langle x, y \mid x^{2}=y^{5}=1\right\rangle \rightarrow P S L(2, \mathbb{C})$ given by $x \mapsto A, y \mapsto B$, then

$$
\begin{aligned}
& \operatorname{tr} \rho\left(x y^{\gamma_{1}} \ldots x y^{\gamma_{\ell}}\right)=\sigma\left(z-\sqrt{4-\eta^{2}}\right) \\
& =\eta^{k_{2}}\left(z-\sqrt{4-\eta^{2}}\right)^{s}\left(z^{2}-2 z \sqrt{4-\eta^{2}}+\eta^{-2}\right)^{t} \\
& \cdot\left(z^{2}-2 z \sqrt{4-\eta^{2}}+1\right)^{u}\left(z-2 \sqrt{4-\eta^{2}}\right)^{v} z^{v}
\end{aligned}
$$

whose constant term is 0 if $v>0$ and is $\eta^{k_{2}-2 t}\left(\sqrt{4-\eta^{2}}\right)^{s}$ if $v=0$. Now the constant term in $\operatorname{tr}\left(A B^{\gamma_{1}} \ldots A B^{\gamma_{\ell}}\right)$ is $2 \cos \left(\left(5 \ell+2 \sum_{i=1}^{\ell} \gamma_{i}\right) \pi / 10\right)$. Since $\ell$ is odd and $\bar{G}$ admits no essential cyclic representation, this constant term is either $\pm 2 \cos (\pi / 10)=$ $\pm \eta \sqrt{4-\eta^{2}}$ or $\pm 2 \cos (3 \pi / 10)= \pm \sqrt{4-\eta^{2}}$. Thus we can conclude that $v=0$, that

$$
\eta^{k_{2}-2 t}\left(\sqrt{4-\eta^{2}}\right)^{s}=\eta \sqrt{4-\eta^{2}} \text { or } \sqrt{4-\eta^{2}}
$$

and therefore that $s=1$ and $t=k_{2} / 2$ or $t=\left(k_{2}-1\right) / 2$. Hence 0 is not a repeated root of $\sigma(\mu)$, contrary to hypothesis.

For the proof of Theorem 2 we shall require the following proposition.

Proposition 11 Let $p \neq q$ be prime numbers, and let $1 \leq t \leq p q-1$. Then

$$
\prod_{\psi \in \operatorname{Aut}\left(\mathbb{Z}_{p q}\right)} 2 \sin \left(\frac{\psi(t) \pi}{p q}\right)= \begin{cases}q^{p-1} & \text { if } p \mid t \\ p^{q-1} & \text { if } q \mid t \\ 1 & \text { otherwise }\end{cases}
$$

\section{Proof}

By identity 1.392(1) of [11] we have that for all real numbers $x$ and $n \geq 2$

$$
\sin (x) \prod_{1 \leq r<n} 2 \sin (x+r \pi / n)=\sin (n x) .
$$

Differentiating and substituting $x=0$ we obtain

$$
\prod_{1 \leq r<n} 2 \sin \left(\frac{r \pi}{n}\right)=n
$$


We now claim that the identity

$$
\prod_{\substack{1 \leq r<n \\(r, n)=1}} 2 \sin \left(\frac{r \pi}{n}\right)= \begin{cases}u & \text { if } n \text { is a power of a prime } u \\ 1 & \text { otherwise }\end{cases}
$$

holds for all $n \geq 2$. This clearly holds when $n=2$. Let $N \geq 3$ and suppose inductively that it holds for all $n<N$. Now

$$
\prod_{1 \leq r<N} 2 \sin \left(\frac{r \pi}{N}\right)=\prod_{\substack{1 \leq r<N \\(r, N)=1}} 2 \sin \left(\frac{r \pi}{N}\right) \cdot \prod_{\substack{d \mid N \\ d>1}} \prod_{\substack{1 \leq r<N \\(r, N)=d}} 2 \sin \left(\frac{r \pi}{N}\right) .
$$

Now

$$
\prod_{\substack{d \mid N \\ d>1}} \prod_{\substack{1 \leq r<N \\(r, N)=d}} 2 \sin \left(\frac{r \pi}{N}\right)=\prod_{\substack{d \mid N \\ d>1}} \prod_{\substack{1 \leq s<N / d \\(s, N / d)=1}} 2 \sin \left(\frac{s \pi}{N / d}\right) .
$$

Applying the inductive hypothesis, the right hand side of (5) is equal to the product of all primes $u$ such that $N / d$ is a power of $u$, where $d>1$ ranges over all divisors of $N$. Thus

$$
\prod_{\substack{d \mid N \\ d>1}} \prod_{\substack{1 \leq r<N \\(r, N)=d}} 2 \sin \left(\frac{r \pi}{N}\right)= \begin{cases}u^{\alpha-1} & \text { if } N=u^{\alpha}, \text { where } \alpha \geq 1 \text { and } u \text { is prime } \\ N & \text { otherwise }\end{cases}
$$

Substituting this into (4) and applying (2) to the left hand side we get that the identity (3) holds for $n=N$ and hence for all $n \geq 2$. Finally,

$$
\begin{aligned}
\prod_{\psi \in \operatorname{Aut}\left(\mathbb{Z}_{p q}\right)} 2 \sin \left(\frac{\psi(t) \pi}{p q}\right) & =\prod_{\substack{1 \leq \alpha<p q \\
(\alpha, p q)=1}} 2 \sin \left(\frac{\alpha t \pi}{p q}\right) \\
& = \begin{cases}\prod_{\substack{1 \leq \alpha<p q \\
(\alpha, p q)=1}} 2 \sin (\alpha \pi / q)=\left(\prod_{\substack{1 \leq \alpha<q \\
(\alpha, q)=1}} 2 \sin (\alpha \pi / q)\right)^{p-1} & \text { if } p \mid t \\
\prod_{\substack{1 \leq \alpha<p q \\
(\alpha, p q)=1}} 2 \sin (\alpha \pi / p)=\left(\prod_{\substack{1 \leq \alpha<p \\
(\alpha, p)=1}} 2 \sin (\alpha \pi / p)\right)^{q-1} & \text { if } q \mid t \\
\prod_{\substack{1 \leq \alpha<p q \\
(\alpha, p q)=1}} 2 \sin (\alpha \pi / p q) & \text { otherwise }\end{cases}
\end{aligned}
$$

and an application of (3) completes the proof.

\section{Proof of Theorem 2}

We will consider the homomorphic image $\bar{G}$ of $G$ defined by the presentation (1). As explained at the start of this section we will assume that $\bar{w}(x, y)$ is not a proper power and can be freely reduced to the form $\bar{w}(x, y)=x y^{\gamma_{1}} \ldots x y^{\gamma_{\ell}}$ where $1 \leq \gamma_{i} \leq 4$ $(1 \leq i \leq \ell-1), \ell \geq 1$.

By [13, Theorem E] we may assume that $G$ admits no essential cyclic representation, and since $m>5$ Lemma 4 implies that the trace polynomial for $G$ has the form $\tau(\lambda)=c \lambda^{k}$, where

$$
c=\frac{1}{(\sin (\pi / 5 p))^{k}} \prod_{i=1}^{k} \sin \left(\frac{\pi \alpha_{i}}{5 p}\right)
$$


Let $X, Y \in P S L(2, \mathbb{C})$ be elements of orders $2,5 p$ that generate a cyclic subgroup of $P S L(2, \mathbb{C})$. We may assume that

$$
X=\left(\begin{array}{cc}
e^{i \pi / 2} & 0 \\
0 & e^{-i \pi / 2}
\end{array}\right), \quad Y=\left(\begin{array}{cc}
e^{i \pi / 5 p} & 0 \\
0 & e^{-i \pi / 5 p}
\end{array}\right)
$$

so that $\operatorname{tr} X Y=2 \sin (\pi / 5 p)$. Let $\rho:\left\langle x, y \mid x^{2}=y^{5 p}=1\right\rangle \rightarrow P S L(2, \mathbb{C})$ be given by $x \mapsto X, y \mapsto Y$. Then $\operatorname{tr} \rho(w)=\operatorname{tr}\left(X^{k} Y^{a}\right)= \pm 2 \sin (a \pi / 5 p)$, where $a=\sum_{i=1}^{k} \alpha_{i}$. On the other hand $\operatorname{tr} \rho(w)=\tau(2 \sin (\pi / 5 p))=\prod_{i=1}^{k} 2 \sin \left(\alpha_{i} \pi / 5 p\right)$. Thus

$$
2 \sin (a \pi / 5 p)= \pm \prod_{i=1}^{k} 2 \sin \left(\alpha_{i} \pi / 5 p\right)
$$

and hence

$$
\prod_{\psi \in \operatorname{Aut}\left(\mathbb{Z}_{5 p}\right)} 2 \sin (\psi(a) \pi / 5 p)= \pm \prod_{i=1}^{k} \prod_{\psi \in \operatorname{Aut}\left(\mathbb{Z}_{5 p}\right)} 2 \sin \left(\psi\left(\alpha_{i}\right) \pi / 5 p\right) .
$$

Suppose $5 \mid \alpha_{i}$ for some $1 \leq i \leq k$. Then by Proposition $11 p^{4}$ divides the right hand side of (6). If $5 \mid a$ then $\bar{G}$ admits an essential cyclic representation and so $\bar{G}$ (and hence $G$ ) contains a non-abelian free subgroup, by Lemma 9. Thus we may assume 5 \a. Proposition 11 then implies that the left hand side of (6) is either equal to 1 or $5^{p-1}$ and we have a contradiction. Thus $5 \backslash \alpha_{i}$ for any $1 \leq i \leq k$ so the (free product) length of $w(x, y)$ is equal to the (free product) length of $\bar{w}(x, y)$. Hence $\ell=k$, and thus the trace polynomial $\sigma(\mu)$ of $\bar{G}$ is of degree $k \geq 3$.

As explained in the proof of Lemma 10 we may assume that $\sigma(\mu)$ is of the form $\sigma(\mu)=c^{\prime} \mu^{s}\left(\mu^{2}-1\right)^{t}\left(\mu^{2}-\eta^{-2}\right)^{u}$ where $\eta=2 \cos (\pi / 5)$ and $s$ is odd. By Lemma 10 we may assume $s=1$, and by Lemma 8 we may assume $t \leq 1$. The automorphism $\theta$ of $\mathbb{Z}_{5}$ generated by the map $1 \mapsto 2$ yields the alternative presentation $\bar{G}=\left\langle x, y \mid x^{2}=y^{5}=\left(x y^{\theta\left(\beta_{1}\right)} \ldots x y^{\theta\left(\beta_{k}\right)}\right)^{2}=1\right\rangle$. The potential roots \pm 1 and $\pm \eta^{-1}$ for $\sigma$ correspond to essential representations $\bar{G} \rightarrow A_{5}$ that map $x y$ to elements of order 3 or 5 respectively (cf. Remark 5 ). The automorphism $\theta$ has the effect of interchanging these two possibilities. Thus the trace polynomial corresponding to this new presentation has the form $\sigma^{\prime}(\mu)=c^{\prime \prime} \mu^{s}\left(\mu^{2}-\eta^{-2}\right)^{t}\left(\mu^{2}-1\right)^{u}$, for some $c^{\prime \prime}$. By another application of Lemma 8 we may assume $u \leq 1$. Since $k=s+2 t+2 u>1$ we are reduced to the cases $k=3,5$.

If $k=3$ then $G$ contains a non-abelian free subgroup by [14, Theorem 1]. If $k=5$ then $s=t=1$ so $\sigma(\mu)=c^{\prime} \mu\left(\mu^{2}-1\right)\left(\mu^{2}-\eta^{-2}\right)$. A computer search reveals that the only words $w(x, y)$ (up to cyclic permutation, inversion, and automorphisms of $\left.\left\langle y \mid y^{5}=1\right\rangle\right)$ with trace polynomial of that form are $x y x y^{3} x y^{2} x y^{4} x y^{t}$ with $t \in$ $\{1,2\}$. In each case, a GAP [10] calculation shows that $\bar{G}$ has a subgroup of index 11 admitting the free group of rank 2 as a homomorphic image, and hence $G$ contains a non-abelian free subgroup. 


\section{The cases $m=12,20,30,60$}

\section{Proof of Theorem 3}

We shall consider alternative presentations for $G$ :

$$
G=\left\langle x, y \mid x^{2}=y^{m}=\left(x y^{\psi\left(\alpha_{1}\right)} \ldots x y^{\psi\left(\alpha_{k}\right)}\right)^{2}=1\right\rangle
$$

where $\psi$ is an automorphism of $\mathbb{Z}_{m}$. By [14, Theorem 5] we may assume that $k$ is odd. By [13, Theorem E] we may assume that $G$ admits no essential cyclic representation. Since $m>5$, Lemma 4 implies that the trace polynomial for $G$ takes the form $\tau(\lambda)=c \lambda^{k}$ where $c=\left(t_{1}^{k_{1}} \ldots t_{m / 2}^{k_{m / 2}}\right) /(\sin (\pi / m))^{k}$. Let $X, Y \in P S L(2, \mathbb{C})$ have orders 2 and $m$ respectively that generate a cyclic group of order $m$. We may assume $\operatorname{tr}(X Y)=2 \sin (\pi / m)$. Fix $\rho$ to be the representation $\rho:\left\langle x, y \mid x^{2}=y^{m}=1\right\rangle \rightarrow$ $P S L(2, \mathbb{C})$ given by $x \mapsto X, y \mapsto Y$. Then

$$
\operatorname{tr} \rho\left(x y^{\psi\left(\alpha_{1}\right)} \ldots x y^{\psi\left(\alpha_{k}\right)}\right)= \pm 2 \cos (q \pi / m) \quad \text { for some } \quad 1 \leq q<m / 2 .
$$

(Note that if $q=m / 2$ then $\rho$ induces an essential cyclic representation of $G$, contrary to our earlier assumption.) In particular,

$$
-1 \leq \prod_{\psi \in A} \frac{\operatorname{tr} \rho\left(x y^{\psi\left(\alpha_{1}\right)} \ldots x y^{\psi\left(\alpha_{k}\right)}\right)}{2} \leq 1
$$

for any group $A$ of automorphisms of $\mathbb{Z}_{m}$.

Now

$$
\begin{aligned}
\operatorname{tr} \rho\left(x y^{\psi\left(\alpha_{1}\right)} \ldots x y^{\psi\left(\alpha_{k}\right)}\right) & =\tau(2 \sin (\pi / m)) \\
& =2^{k} \prod_{i=1}^{k} \sin \left(\frac{\pi \psi\left(\alpha_{i}\right)}{m}\right)
\end{aligned}
$$

so

$$
\frac{\operatorname{tr} \rho\left(x y^{\psi\left(\alpha_{1}\right)} \ldots x y^{\psi\left(\alpha_{k}\right)}\right)}{2}=2^{k-1} \cdot t_{1}^{k_{\psi(1)}} \ldots t_{m / 2}^{k_{\psi(m / 2)}} .
$$

We now consider each value of $m$ separately.

The case $m=12$.

Let $\psi$ be the automorphism of $\mathbb{Z}_{12}$ generated by the map $1 \mapsto 5$ and let $A=\langle\psi\rangle$. Then using (8) and (9) we obtain

$$
2^{2(k-1)}\left(t_{1} t_{5}\right)^{k_{1}+k_{5}} \cdot\left(t_{2}\right)^{2 k_{2}} \cdot\left(t_{3}\right)^{2 k_{3}} \cdot\left(t_{4}\right)^{2 k_{4}} \cdot\left(t_{6}\right)^{2 k_{6}} \leq 1
$$

which (using (3)) simplifies to

$$
2^{k_{3}+2 k_{6}-2} \cdot 3^{k_{4}} \leq 1
$$


We shall consider the following homomorphic images of $G$ :

$$
\begin{aligned}
H & =\left\langle x, y \mid x^{2}=y^{6}=\left(x y^{\beta_{1}} \ldots x y^{\beta_{k}}\right)^{2}=1\right\rangle, \\
L & =\left\langle x, y \mid x^{2}=y^{4}=\left(x y^{\gamma_{1}} \ldots x y^{\gamma_{k}}\right)^{2}=1\right\rangle,
\end{aligned}
$$

where $\beta_{i}=\alpha_{i} \bmod 6$ and $\gamma_{i}=\alpha_{i} \bmod 4$ for each $1 \leq i \leq k$. Suppose $k_{6}=0$. Then each $\beta_{i}$ is non-zero. If $k>3$ then by Theorem $1 H$, and hence $G$, contains a non-abelian free subgroup. If $k=3$ then by [14, Theorem 1] $G$ contains a non-abelian free subgroup. Thus we may assume $k_{6} \geq 1$ and hence $k_{6}=1, k_{3}=k_{4}=0$. Moreover we may assume

$$
\operatorname{tr} \rho\left(x y^{\alpha_{1}} \ldots x y^{\alpha_{k}}\right)= \pm 2
$$

for otherwise one of $\rho\left(x y^{\alpha_{1}} \ldots x y^{\alpha_{k}}\right)$ or $\rho\left(x y^{\psi\left(\alpha_{1}\right)} \ldots x y^{\psi\left(\alpha_{k}\right)}\right)$ provides a contradiction to (7). Using (9) equation (10) simplifies to

$$
\begin{aligned}
2 & =2^{k_{1}+k_{2}+k_{5}+1} \cdot t_{1}^{k_{1}} t_{2}^{k_{2}} t_{5}^{k_{5}} t_{6}^{1} \\
& =2\left(\frac{\sqrt{6}-\sqrt{2}}{2}\right)^{k_{1}-k_{5}}
\end{aligned}
$$

so $k_{1}=k_{5}$. Since the image of $\rho$ is isomorphic to $\mathbb{Z}_{12}$ and by equation (10) $\rho(w)$ is the zero of this group we have that $6 k+\sum_{i=1}^{k} \alpha_{i}=0 \bmod 12$, and $k$ is odd so

$$
\sum_{i=1}^{k} \alpha_{i}=6 \bmod 12,
$$

which implies $\sum_{i=1}^{k} \gamma_{i}=2 \bmod 4$. By Lemma $6 L$ (and hence $G$ ) contains a nonabelian free subgroup unless precisely one $\gamma_{i}=2$. This implies that $k_{2}+k_{6}=1$, but $k_{6}=1$ so $k_{2}=0$.

Let $\bar{w}(x, y)=x y^{\beta_{1}} \ldots x y^{\beta_{k}}$. Using the relations $x^{2}=1, y^{6}=1$ of $H$ we can cyclically reduce $\bar{w}(x, y)$ to $x$ (in which case $H \cong \mathbb{Z}_{2} * \mathbb{Z}_{6}$, so $G$ contains a non-abelian free subgroup) or to the form $\bar{w}(x, y)=x y^{\delta_{1}} \ldots x y^{\delta_{\ell}}$ where $\ell$ is odd and $1 \leq \delta_{i} \leq 5$ for each $1 \leq i \leq \ell$. If $\ell>3$ then by Theorem $1 H$, and hence $G$, contains a non-abelian free subgroup. Thus we may assume $\ell=1$ or 3 . The words $w, \bar{w}$ then take the following forms:

$$
\begin{array}{lll}
\ell=1: & w=x y^{\xi_{1}} x y^{\xi_{2}} u(x, y) x y^{6} v(x, y) & \bar{w}=x y^{\xi_{1}+\xi_{2}} \\
\ell=3: & w=x y^{\xi_{1}} x y^{\xi_{2}} x y^{\xi_{3}} x y^{\xi_{4}} u(x, y) x y^{6} v(x, y) & \bar{w}=x y^{\xi_{1}+\xi_{4}} x y^{\xi_{2}} x y^{\xi_{3}}
\end{array}
$$

where $\xi_{1}, \xi_{2}, \xi_{3}, \xi_{4} \in\{1,5\}$ and

$$
\begin{gathered}
u(x, y)=x y^{a_{1}} \ldots x y^{a_{n}}, \\
v(x, y)=x y^{b_{n}} \ldots x y^{b_{1}},
\end{gathered}
$$

with $a_{i}+b_{i}=0 \bmod 6$ for each $1 \leq i \leq n$. 
In the case $\ell=1$ equation (11) implies $\sum_{i=1}^{k} \alpha_{i}=0 \bmod 6$ so

$$
\xi_{1}+\xi_{2}+\left(a_{1}+\cdots+a_{n}\right)+6+\left(b_{n}+\cdots+b_{1}\right)=0 \bmod 6
$$

which implies $\xi_{1}+\xi_{2}=0$ mod 6 contradicting our assumption that the exponents of $y$ in $\bar{w}$ are non-zero. In the case $\ell=3$, since $\xi_{1}+\xi_{2}+\xi_{3}+\xi_{4}$ is even, Theorem 1 of [14] implies that $H$, and hence $G$, contains a non-abelian free subgroup.

The case $m=20$.

We shall consider the following homomorphic image of $G$ :

$$
H=\left\langle x, y \mid x^{2}=y^{10}=\left(x y^{\beta_{1}} \ldots x y^{\beta_{k}}\right)^{2}=1\right\rangle
$$

where $\beta_{i}=\alpha_{i} \bmod 10$ for each $1 \leq i \leq k$.

Let $\psi$ be the automorphism of $\mathbb{Z}_{20}$ generated by the map $1 \mapsto 3$ and let $A=\langle\psi\rangle$. Then using (8) and (9) we obtain

$$
2^{4(k-1)}\left(t_{1} t_{3} t_{7} t_{9}\right)^{k_{1}+k_{3}+k_{7}+k_{9}}\left(t_{2} t_{6}\right)^{2\left(k_{2}+k_{6}\right)}\left(t_{4} t_{8}\right)^{2\left(k_{4}+k_{8}\right)} t_{5}^{4 k_{5}} t_{10}^{4 k_{10}} \leq 1
$$

which (using (3)) simplifies to

$$
2^{2 k_{5}+4 k_{10}-4} \cdot 5^{k_{4}+k_{8}} \leq 1
$$

If $k_{10}=0$ then each $\beta_{i}$ is non-zero so $H$ contains a non-abelian free subgroup by Theorem 2. Thus we may assume that $k_{10} \geq 1$ and hence $k_{10}=1, k_{5}=k_{4}=k_{8}=0$. Moreover we may assume

$$
\operatorname{tr} \rho\left(x y^{\alpha_{1}} \ldots x y^{\alpha_{k}}\right)= \pm 2
$$

for otherwise for some $\phi \in A$ the element $\rho\left(x y^{\phi\left(\alpha_{1}\right)} \ldots x y^{\phi\left(\alpha_{k}\right)}\right)$ provides a contradiction to (7). The image of $\rho$ is isomorphic to $\mathbb{Z}_{20}$ and by equation (12) $\rho(w)$ is the zero of this group so we have that $\sum_{i=1}^{k} \alpha_{i}=10 \bmod 20$ (since $k$ is odd). Thus $\sum_{i=1}^{k} \beta_{i}=0 \bmod 10$ so $H$ admits an essential cyclic representation, and the result follows from [13, Theorem E].

The case $m=30$.

We shall consider the following homomorphic images of $G$ :

$$
\begin{aligned}
H & =\left\langle x, y \mid x^{2}=y^{10}=\left(x y^{\beta_{1}} \ldots x y^{\beta_{k}}\right)^{2}=1\right\rangle, \\
L & =\left\langle x, y \mid x^{2}=y^{15}=\left(x y^{\gamma_{1}} \ldots x y^{\gamma_{k}}\right)^{2}=1\right\rangle,
\end{aligned}
$$

where $\beta_{i}=\alpha_{i} \bmod 10, \gamma_{i}=\alpha_{i} \bmod 15$ for each $1 \leq i \leq k$.

Let $\psi$ be the automorphism of $\mathbb{Z}_{30}$ generated by the map $1 \mapsto 7$ and let $A=\langle\psi\rangle$. Then using (8) and (9) we obtain

$$
\begin{aligned}
& 2^{4(k-1)}\left(t_{1} t_{7} t_{11} t_{13}\right)^{k_{1}+k_{7}+k_{11}+k_{13}}\left(t_{2} t_{14} t_{8} t_{4}\right)^{k_{2}+k_{14}+k_{8}+k_{4}} \\
& \cdot\left(t_{3} t_{9}\right)^{2\left(k_{3}+k_{9}\right)}\left(t_{5}\right)^{4 k_{5}}\left(t_{6} t_{12}\right)^{2\left(k_{6}+k_{12}\right)} t_{10}^{4 k_{10}} t_{15}^{4 k_{15}} \\
& \leq 1
\end{aligned}
$$


which (using (3)) simplifies to

$$
2^{4 k_{15}-4} \cdot 5^{k_{6}+k_{12}} \cdot 9^{k_{10}} \leq 1 .
$$

If $k_{15}=0$ then each $\gamma_{i}$ is non-zero which implies that $L$, and hence $G$, contains a non-abelian free subgroup by Theorem 2 . If $k_{15}>0$ then $k_{10}=0$, so $H$, and hence $G$, contains a non-abelian free subgroup by Theorem 2 .

The case $m=60$.

We shall consider the following homomorphic images of $G$ :

$$
\begin{aligned}
H & =\left\langle x, y \mid x^{2}=y^{20}=\left(x y^{\beta_{1}} \ldots x y^{\beta_{k}}\right)^{2}=1\right\rangle, \\
L & =\left\langle x, y \mid x^{2}=y^{30}=\left(x y^{\gamma_{1}} \ldots x y^{\gamma_{k}}\right)^{2}=1\right\rangle,
\end{aligned}
$$

where $\beta_{i}=\alpha_{i} \bmod 20, \gamma_{i}=\alpha_{i} \bmod 30$ for each $1 \leq i \leq k$.

Consider the group $A \cong \mathbb{Z}_{4} \times \mathbb{Z}_{2}$ of automorphisms of $\mathbb{Z}_{60}$ generated by $\psi: 1 \mapsto 7$ and $\phi: 1 \mapsto 29$. Using (8) and (9) we obtain

$$
\begin{aligned}
1 \geq & 2^{8(k-1)} \\
& \cdot\left(t_{1} t_{7} t_{11} t_{13} t_{17} t_{19} t_{23} t_{29}\right)^{k_{1}+k_{7}+k_{11}+k_{13}+k_{17}+k_{19}+k_{23}+k_{29}} \\
& \cdot\left(t_{2} t_{14} t_{22} t_{26}\right)^{2\left(k_{2}+k_{14}+k_{22}+k_{26}\right)} \cdot\left(t_{3} t_{21} t_{27} t_{9}\right)^{2\left(k_{3}+k_{21}+k_{27}+k_{9}\right)} \\
& \cdot\left(t_{4} t_{28} t_{16} t_{8}\right)^{2\left(k_{4}+k_{28}+k_{16}+k_{8}\right)} \cdot\left(t_{5} t_{25}\right)^{4\left(k_{5}+k_{25}\right)} \cdot\left(t_{6} t_{18}\right)^{4\left(k_{6}+k_{18}\right)} \cdot\left(t_{12} t_{24}\right)^{4\left(k_{12}+k_{24}\right)} \\
& \cdot\left(t_{10}\right)^{8 k_{10}} \cdot\left(t_{15}\right)^{8 k_{15}} \cdot\left(t_{20}\right)^{8 k_{20}} \cdot\left(t_{30}\right)^{8 k_{30}}
\end{aligned}
$$

which (using (3)) simplifies to

$$
1 \geq 2^{4 k_{15}+8 k_{30}-8} \cdot 5^{2\left(k_{12}+k_{24}\right)} \cdot 3^{4 k_{20}}
$$

In particular one of $k_{20}, k_{30}$ is zero so either all $\beta_{i}$ 's are non-zero or all $\gamma_{i}$ 's are nonzero. Hence, by the above, one of $H$ or $L$ (and hence $G$ ) contains a non-abelian free subgroup.

\section{A Appendix: The case $m=6$}

This appendix gives a sketch proof of Theorem 1. We begin by giving a complete calculation of all the coefficients of the trace polynomial.

Let $\mathcal{A}(k)$ denote the set of subsets $S \subset\{1, \ldots, k\}$ such that $s_{1}-s_{2} \neq 1(\bmod k)$ for $s_{1}, s_{2} \in S$. The maximum cardinality of $S \in \mathcal{A}(k)$ is the integer part $\lfloor k / 2\rfloor$ of $k / 2$. For $0 \leq j \leq\lfloor k / 2\rfloor$, let $\mathcal{A}(k, j)$ denote the set of sets $S \in \mathcal{A}(k)$ of cardinality $j$.

Lemma 12 Let $X, Y \in S L(2, \mathbb{C})$ be matrices with $\operatorname{tr}(X)=0, \operatorname{tr}(Y)=2 \cos (\pi / m)$, $\operatorname{tr}(X Y)=\lambda$, for some integer $m \geq 2$. Let $W=X Y^{\alpha_{1}} \ldots X Y^{\alpha_{k}}$, where $1 \leq \alpha_{i}<m$ for each $1 \leq i \leq k$. Then the trace of $W$ is given by the polynomial

$$
\operatorname{tr}(W)=c \sum_{j=0}^{\lfloor k / 2\rfloor}(-1)^{j} B_{j} \lambda^{k-2 j},
$$


where

$$
\begin{aligned}
c & =\prod_{j=1}^{k} \frac{\sin \left(\alpha_{j} \pi / m\right)}{\sin (\pi / m)}, \\
B_{j} & =\sum_{\left\{t_{1}, \ldots, t_{j}\right\} \in \mathcal{A}(k, j)}\left(\prod_{s=1}^{j} b\left(t_{s}\right)\right), \\
b(j) & =\frac{\sin ^{2}(\pi / m) e^{i \pi\left(\alpha_{j+1}-\alpha_{j}\right) / m}}{\sin \left(\alpha_{j} \pi / m\right) \sin \left(\alpha_{j+1} \pi / m\right)} .
\end{aligned}
$$

\section{Proof}

By [12] the trace of $W(X, Y)$ is determined by the traces of $X, Y$ and $X Y$, so it is sufficient to work with fixed matrices with the given traces. We define

$$
X=\left(\begin{array}{cc}
0 & -1 \\
1 & 0
\end{array}\right), \quad Y=\left(\begin{array}{cc}
e^{i \pi / m} & \lambda \\
0 & e^{-i \pi / m}
\end{array}\right)
$$

Then, for $1 \leq \alpha \leq m-1$,

$$
X Y^{\alpha}=\left(\begin{array}{cc}
0 & -e^{-i \alpha \pi / m} \\
e^{i \alpha \pi / m} & p(\alpha) \lambda
\end{array}\right)
$$

with $p(\alpha)=\sin (\alpha \pi / m) / \sin (\pi / m)$. Now each entry in $W(X, Y)$ is a sum of terms, each of which is a product of an entry from each of $X Y^{\alpha_{j}}(1 \leq j \leq k)$. The leading monomial of $\operatorname{tr}(W(X, Y))$ necessarily consists of the product of the lower right entries of the $X Y^{\alpha_{j}}$, so is $c \lambda^{k}=\prod_{j=1}^{k} p\left(\alpha_{j}\right) \lambda^{k}$, as claimed. Each term contributing to the $\lambda^{k-2 j}$ monomial can be obtained from $c$ by replacing each of $j$ (non-overlapping) pairs of (cyclically) consecutive lower right entries by the upper right entry of the first member of the pair, followed by the lower left entry of the second member. Such a term is thus equal to $c b\left(s_{1}\right) \cdots b\left(s_{j}\right)$ for some $\left\{s_{1}, \ldots, s_{j}\right\} \in \mathcal{A}(k, j)$, and the result follows.

\section{Sketch proof of Theorem 1}

Let

$$
\begin{aligned}
& G=\left\langle x, y \mid x^{2}=y^{6}=w(x, y)^{2}=1\right\rangle, \\
& \bar{G}=\left\langle x, y \mid x^{2}=y^{3}=\bar{w}(x, y)^{2}=1\right\rangle,
\end{aligned}
$$

where $w(x, y)=x y^{\alpha_{1}} \ldots x y^{\alpha_{k}}, \bar{w}(x, y)=x y^{\beta_{1}} \ldots x y^{\beta_{k}}$ where for $1 \leq i \leq k, \beta_{i}=$ $\alpha_{i} \bmod 3$, and $k>3$. Let $\tau(\lambda), \sigma(\mu)$ denote the trace polynomials of $G, \bar{G}$ respectively. By Lemma 4 if $G$ contains no non-abelian free subgroup then the roots of $\tau$ are among 0 , corresponding to an essential representation onto the dihedral group $D_{12}$, or \pm 1 , which occur if and only if $G$ admits an essential cyclic representation.

Suppose first that $G$ admits an essential cyclic representation, with kernel $K$. Then \pm 1 are roots of $\tau(\lambda)$. By [13, Theorem 4.8] if 1 or -1 is a repeated root of 
$\tau(\lambda)$ then $G$ has a non-abelian free subgroup. Thus we may assume that $\tau(\lambda)=$ $c \lambda^{k-2}\left(\lambda^{2}-1\right)$ and in particular that $G$ has an essential representation $\rho$ onto $D_{12}$. Now $K$ has a deficiency 0 presentation, its abelianization $K / K^{\prime}$ is free abelian of rank 3 , and conjugation by $x$ induces the antipodal automorphism on $K / K^{\prime}$. Moreover, a calculation shows that $\rho\left(K^{\prime}\right)$ is a non-trivial abelian subgroup of $D_{12}$, so $K^{\prime} / K^{\prime \prime}$ is non-trivial. By [13, Corollary 3.2], $K^{\prime}$ (and hence $G$ ) contains a non-abelian free subgroup.

Hence we may assume that $G$ has no essential cyclic representations, and thus $\tau(\lambda)=c \lambda^{k}$. Then as in the proof of Theorem 3 equations (8), (9) yield $\left(k_{2}, k_{3}\right)=$ $(0,0),(1,0),(0,1)$ and thus $c=1, \sqrt{3}, 2$, respectively. When $k$ is even the existence of an essential dihedral representation implies that the alternating sum $\sum_{i=1}^{k}(-1)^{i} \alpha_{i}$ is congruent to 3 modulo 6 and thus $k_{2}=1, c=\sqrt{3}$.

We proceed by calculating the coefficients in $\tau(\lambda), \sigma(\mu)$ and split the proof into three cases, depending on the value of $c$. Consider first the form of $\sigma(\mu)$ in the cases $c=1, \sqrt{3}$. By Lemma 4 and Remark 5 we may assume that the roots of $\sigma$ are among $\pm 1, \pm \sqrt{2},( \pm 1 \pm \sqrt{5}) / 2, \pm \sqrt{3}, 0$. If \pm 1 or $\pm \sqrt{3}$ occurs as a root of $\sigma$ then $\bar{G}$ admits an essential representation to $A_{4}$ or $\mathbb{Z}_{6}$. In either case $\sum_{i=1}^{k} \beta_{i}=0 \bmod 3$, and we can define a representation $\rho: G \rightarrow \mathbb{Z}_{6}$ by $\rho(x)=3 \bmod 6$ and $\rho(y)=1 \bmod 6$. By assumption, $\rho$ is not essential, so $\rho(w)=0 \bmod 6$ and $c=\tau(1)= \pm 2$, a contradiction. Since $\sigma$ has rational coefficients we thus have

$$
\sigma(\mu)=\mu^{r}\left(\mu^{2}-2\right)^{s}\left(\mu^{4}-3 \mu^{2}+1\right)^{t}
$$

where $r, s, t \geq 0$ satisfy $r+2 s+4 t=k$. Since $\sigma(\sqrt{3}) \in\{ \pm 1, \pm \sqrt{3}, \pm 2\}$ we have $r=0,1$. If $k$ is even then $r=0$, and (since $\sum_{i=1}^{k}(-1)^{i} \alpha_{i}$ is congruent to 0 modulo 3 ) we also have $\sigma(0)= \pm 2$ so $s=1$.

Case 1: $c=1$.

In this case $k$ is odd and $\alpha_{i} \in\{1,5\}$ for each $1 \leq i \leq k$. By Lemma 12, the coefficient $-B_{1}$ of $\lambda^{k-2}$ in $\tau(\lambda)$ is given by $B_{1}=\sum_{i=1}^{k} b(i)$, where for each $1 \leq i \leq k$

$$
b(i):= \begin{cases}1 & \text { if } \alpha_{i}=\alpha_{i+1} \\ \frac{-1+\sqrt{-3}}{2} & \text { if } \alpha_{i}=1, \alpha_{i+1}=5 \\ \frac{-1-\sqrt{-3}}{2} & \text { if } \alpha_{i}=5, \alpha_{i+1}=1\end{cases}
$$

(where $\alpha_{k+1}$ is defined equal to $\alpha_{1}$ ). A similar analysis for $\sigma(\mu)$ shows that the coefficient $-B_{1}^{\prime}$ of $\mu^{k-2}$ is given by $B_{1}^{\prime}=\sum_{i=1}^{k} b^{\prime}(i)$ where

$$
b^{\prime}(i):= \begin{cases}1 & \text { if } \beta_{i}=\beta_{i+1} \\ \frac{1+\sqrt{-3}}{2} & \text { if } \beta_{i}=1, \beta_{i+1}=2 \\ \frac{1-\sqrt{-3}}{2} & \text { if } \beta_{i}=2, \beta_{i+1}=1\end{cases}
$$

Since the coefficient of $\lambda^{k-2}$ in $\tau(\lambda)$ is zero, we have that $k$ is a multiple of 3 - say $k=3 \ell$ where $\ell>1$ - and each possible value of $b(i)$ occurs precisely $\ell$ times. It follows that $B_{1}^{\prime}=2 \ell$. On the other hand we can compute the coefficient of $\mu^{k-2}$ 
in $\sigma(\mu)=\mu\left(\mu^{2}-2\right)^{s}\left(\mu^{4}-3 \mu^{2}+1\right)^{t}$ as $-2 s-3 t$. We thus obtain the simultaneous diophantine equations

$$
1+2 s+4 t=3 \ell, \quad 2 s+3 t=2 \ell, \quad s, t, \geq 0, \ell>1
$$

with the unique solution $s=0, t=2, \ell=3$, and so $k=9$.

Now consider the coefficient $B_{2}$ of $\lambda^{5}$ in $\tau(\lambda)$ and the coefficient $B_{2}^{\prime}$ of $\mu^{5}$ in $\sigma(\mu)$. Using Lemma 12 we can deduce

$$
2 B_{2}=B_{1}^{2}-\sum_{i=1}^{9} b(i)^{2}-2 \sum_{i=1}^{9} b(i) b(i+1)
$$

where $b(10)$ is defined equal to $b(1)$. Since $B_{1}=B_{2}=0$ and the $b(i)$ 's are equally distributed amongst the three possible values it follows that $\sum_{i=1}^{9} b(i) b(i+1)=0$.

A similar analysis shows that $\sum_{i=1}^{9} b^{\prime}(i)^{2}=0, \sum_{i=1}^{9} b^{\prime}(i) b^{\prime}(i+1)=6$, from which we can deduce $B_{2}^{\prime}=12$. But the coefficient of $\mu^{5}$ in $\sigma(\mu)=\mu\left(\mu^{4}-3 \mu^{2}+1\right)^{2}$ is 11 . This contradiction completes Case 1 .

Case 2: $c=\sqrt{3}$.

Then $\alpha_{i} \in\{1,5\}$ for all but one value of $i$, for which $\alpha_{i} \in\{2,4\}$. Without loss of generality we may assume that $\alpha_{k}=2$ and $\alpha_{i} \in\{1,5\}$ for $1 \leq i<k$. As in Case 1 , consideration of the coefficient of $\lambda^{k-2}$ in $\tau(\lambda)$ and of $\mu^{k-2}$ in $\sigma(\mu)$ yield diophantine equations in $s, t, k$. We find that the only solutions with $k>3$ are (i) $s=2, t=0$, $k=5$; (ii) $s=0, t=2, k=9$; (iii) $s=1, t=2, k=11$; (iv) $s=0, t=4, k=17$; (v) $s=0, t=2, k=8$. We can rule out solution (v) since $k$ is even and $s \neq 1$.

For the remaining solutions, consideration of the coefficient of $\lambda^{k-4}$ in $\tau(\lambda)$ and the coefficient of $\mu^{k-4}$ in $\sigma(\mu)$ yield additional diophantine equations which reduce us to solution (i). A computer search reveals that the only word $w(x, y)$ (up to cyclic permutation, inversion, and automorphisms of $\left\langle y \mid y^{6}=1\right\rangle$ ) such that $\tau(\lambda), \sigma(\mu)$ are of the required form is $w(x, y)=x y^{5} x y x y x y^{5} x y^{2}$. A calculation in GAP [10] shows that in this case $G$ has a subgroup of index 6 admitting a free homomorphic image of rank 2 .

Case 3: $c=2$.

In this case $k$ is odd, the $\alpha_{i}$ are all odd, and $\alpha_{i}=3$ for precisely one value of $i$. Without loss of generality we may assume that $\alpha_{k}=3$ and $\alpha_{i} \in\{1,5\}$ for $1 \leq i<k$. Again, the coefficient $-B_{1}$ of $\lambda^{k-2}$ is given by $B_{1}=\sum_{i=1}^{k} b(i)$ where $b(i)$ is as in Case 1 for $i<k-1$,

$$
b(k-1):= \begin{cases}\frac{1+\sqrt{-3}}{4} & \text { if } \alpha_{k-1}=1 \\ \frac{1-\sqrt{-3}}{4} & \text { if } \alpha_{k-1}=5\end{cases}
$$

and

$$
b(k):= \begin{cases}\frac{1-\sqrt{-3}}{4} & \text { if } \alpha_{1}=1 \\ \frac{1+\sqrt{-3}}{4} & \text { if } \alpha_{1}=5\end{cases}
$$


Note that $b(1), \ldots, b(k-2)$ are algebraic integers. From the equation $B_{1}=0$ it follows that $b(k-1)+b(k)$ is also an algebraic integer, and this can only happen if $\alpha_{1}+\alpha_{k-1}=6$. Assume inductively that $\alpha_{t}+\alpha_{k-t}=6$ (and hence $b(k-t)=b(t-1)$, where $b(0)$ is defined equal to $b(k))$ for $1 \leq t<u$, for some $u \leq(k-1) / 2$. Then from the equation $B_{u}=0$ it turns out that $b(k-u)+b(u-1)$ is an algebraic integer, and this can only happen if $\alpha_{u}+\alpha_{k-u}=6$.

Thus $\alpha_{t}+\alpha_{k-t}=6$ for all $1 \leq t \leq(k-1) / 2$, so the third relator of $G$ has the form $\left(U(x, y) x U(x, y)^{-1} y^{3}\right)^{2}$ for some word $U$. In passing to $\bar{G}$, we kill $y^{3}$, so the relator collapses to $x^{2}$, and $\bar{G} \cong \mathbb{Z}_{2} * \mathbb{Z}_{3}$. Hence $\bar{G}$, and so also $G$, contains a non-abelian free subgroup, as claimed.

\section{References}

[1] O.A. Barkovich and V.V. Benyash-Krivets. On Tits alternative for generalized triangular groups of $(2,6,2)$ type (Russian). Dokl. Nat. Akad. Nauk. Belarusi, 48(3):28-33, 2003.

[2] Gilbert Baumslag, John W. Morgan, and Peter B. Shalen. Generalized triangle groups. Math. Proc. Cambridge Philos. Soc., 102(1):25-31, 1987.

[3] V.V. Benyash-Krivets. On free subgroups of certain generalised triangle groups (Russian). Dokl. Nat. Akad. Nauk. Belarusi, 47(3):14-17, 2003.

[4] V.V. Benyash-Krivets. On Rosenberger's conjecture for generalized triangle groups of types $(2,10,2)$ and $(2,20,2)$. In Shyam L. Kalla et al., editor, Proceedings of the international conference on mathematics and its applications, pages 59-74. Kuwait Foundation for the Advancement of Sciences, 2005.

[5] V.V Benyash-Krivets and O.A. Barkovich. On the Tits alternative for some generalized triangle groups. Algebra Discrete Math., 2004(2):23-43, 2004.

[6] Robert Bieri and Ralph Strebel. Valuations and finitely presented metabelian groups. Proc. London Math. Soc. (3), 41(3):439-464, 1980.

[7] H.S.M. Coxeter and W.O.J. Moser. Generators and relations for discrete groups. Ergeb. Math. Grenzgebiette. Springer-Verlag, Berlin-Heidelberg-New York, 1972.

[8] Benjamin Fine, Frank Levin, and Gerhard Rosenberger. Free subgroups and decompositions of one-relator products of cyclics. I. The Tits alternative. Arch. Math. (Basel), 50(2):97-109, 1988.

[9] Benjamin Fine, Frank Roehl, and Gerhard Rosenberger. The Tits alternative for generalized triangle groups. In Young Gheel et al. Baik, editor, Groups - Korea '98. Proceedings of the 4th international conference, Pusan, Korea, August 10-16, 1998., pages 95-131. Berlin: Walter de Gruyter., 2000. 
[10] The GAP Group. GAP - Groups, Algorithms, and Programming, Version 4.4, 2004. (http://www.gap-system.org).

[11] I. S. Gradshteyn and I. M. Ryzhik. Table of integrals, series, and products. Academic Press Inc., Boston, MA, fifth edition, 1994. Translation edited and with a preface by Alan Jeffrey.

[12] Robert D. Horowitz. Characters of free groups represented in the twodimensional special linear group. Comm. Pure Appl. Math., 25:635-649, 1972.

[13] James Howie. Free subgroups in groups of small deficiency. J. Group Theory, 1(1):95-112, 1998.

[14] Frank Levin and Gerhard Rosenberger. On free subgroups of generalized triangle groups. II. In Group theory (Granville, OH, 1992), pages 206-228. World Sci. Publishing, River Edge, NJ, 1993.

[15] Gerhard Rosenberger. On free subgroups of generalized triangle groups. Algebra i Logika, 28(2):227-240, 245, 1989.

[16] Alun G.T. Williams. Generalised triangle groups of type $(2, m, 2)$. In M. Atkinson et al, editor, Computational and Geometric Aspects of Modern Algebra, LMS Lecture Note Series 275, pages 265-279. Cambridge University Press, 2000.

\author{
Author addresses: \\ James Howie \\ School of Mathematical and Computer Sciences \\ Heriot-Watt University \\ Edinburgh EH14 4AS \\ J.Howie@hw.ac.uk \\ Gerald Williams \\ Institute of Mathematics, Statistics and Actuarial Science \\ University of Kent \\ Canterbury \\ Kent CT2 7NF \\ g.williams@kent.ac.uk
}

